\title{
Entre la escolarización y el sentido de educar en Psicopedagogía: una reflexión
}

\author{
Between schooling and the meaning of education in Psychopedagogy: a reflection
}

Recibido 10 mayo 2015 • Aceptado 28 mayo 2015 • Corregido 31 agosto 2015

\author{
Rodrigo Alberto Espinoza Vásquez ${ }^{1}$ \\ Psicopedagogo \\ Profesor de Educación Diferencial mención TEA \\ La Serena, Chile \\ respinoza.psicopedagogia@gmail.com
}

\begin{abstract}
Resumen. En el presente trabajo se aborda la temática de la disposición epistemológica y legal de los profesionales psicopedagogos de la nación chilena a partir de lo propuesto por el decreto 170 en el que se generan limitantes sobre el rol, posterior al desarrollo de la evaluación diagnóstica conocida como Intervención Psicopedagógica. El texto parte desde el cuestionamiento y confusión entre los términos educación y escolarización, utilizados como sinónimos, y su relación con la Psicopedagogía como profesión de especialidad para aquellos niños y niñas que se ven enfrentados a barreras en su aprendizaje. Se analizan las acciones de mediación y se presenta el término acompañamiento por el utilizado comúnmente: intervención, pues recoge una disposición emocional diferente y vinculante con las estudiantes y los estudiantes. Finalmente se propone la idea de que la Psicopedagogía surge desde un espacio escolarizado, pero que progresivamente puede avanzar desde este hacia una educación liberadora, pudiendo significar una alternativa paradigmática en virtud de lo propuesto por el decreto en cuestión, que se basa en una perspectiva médico-jurídica.
\end{abstract}

Palabras clave: Psicopedagogía; escolarizar; patologizar; educación; acompañamiento.

Abstract. In the present paper deals with the theme of the layout epistemological and legal of the professional school counselors (learning disabilities) in Chile from that proposed by the decree 170 in which they were produced limitations on the role, subsequent to the development of the diagnostic evaluation known as educational psychopedagogycal intervention. The text part from the questioning and confusion between the terms education and schooling, used as synonyms, and its relationship with the Psychopedagogy as a profession of specialty for those children who are confronted with barriers in their learning. Examining the actions of mediation and presents the term accompaniment by the commonly used: intervention, as it contains a provision different emotional

1 Magister en Gestión y Liderazgo Educativo de la Universidad Central de Chile. Licenciado en Educación de la Universidad de Playa Ancha de Ciencias de la Educación (UPLA). Profesor de Educación Diferencial con mención en Trastornos del Aprendizaje de la Universidad de Playa Ancha de Ciencias de la Educación y Psicopedagogo del Instituto Profesional Los Leones. Actualmente es profesor de la Universidad Central de Chile, Universidad Santo Tomás, Instituto Profesional de Chile e Instituto Profesional La Araucana y Asesor Técnico de la Corporación Servicio de Paz y Justicia (SERPAJ-Chile). 
and binding with the learner. Finally, it is proposed the idea that educational psychology arises from a space school, but that can then progressively move from this toward a liberating education, which may mean an alternative paradigmatic in virtue of what is proposed by the decree in question, which is based on a medical-legal perspective.

Keywords: Psychopedagogy; schooling; pathologizing; education; accompaniment.

\section{Un contexto dialógico}

En una conversación con una de las autoras ${ }^{2}$ del decreto 170 cuyo objetivo es fijar nuevas disposiciones en las subvenciones de estudiantes que son categorizados según las necesidades educativas especiales (NEE), a partir de evaluaciones diagnósticas médico-pedagógicas, se le hizo notar la incongruencia que existía en la descripción del trabajo psicopedagógico, esto es: evaluar, pero no acompañar en el proceso posterior a las personas estudiantes, desde una disposición cognitiva, instrumental y socioafectiva por parte del profesional. La aludida dejó en manifiesto que los psicopedagogos no se encuentran preparados para trabajar en colegios, pues las mallas curriculares de las instituciones de educación superior no están diseñadas ni intencionadas para ello. Frente a esto, el argumento que se contrapone a esta idea es que no es factible, ya que la Psicopedagogía en Chile está diseñada para trabajar con niñas y niños en un contexto de escuela.

\section{El sentido de la escolarización y la educación}

La Psicopedagogía en Chile es tributaria de los modelos españoles, que en su gran medida trabaja con niñas y niños escolarizados. En nuestro país, la intención formadora de nuevos profesionales escasamente se ha acercado a otras dimensiones, entre ellas: el trabajo sociocomunitario. La escolarización y la Psicopedagogía están tan enraizadas y conviven en una relación simbiótica, que son parte de una misma estructura, volviéndose una especie de ortopedia escolar, que asume como propios los fracasos que el estudiantado y el cuerpo docente van teniendo en su recorrido escolar.

Frente a esto, surge una pregunta que podría abrir las puertas a una mirada diferente de la disciplina. ¿Es acaso la Psicopedagogía una suma de acciones tendientes a escolarizar o a vivir la educación de una manera diversa?, ¿en qué momentos se opta por reescolarizar o por educar?

La profesión sigue viviendo aún bajo paradigmas positivistas, aunque se intente un cambio a nivel de formación y se hable de constructivismo, comunicando hasta el cansancio los planteamientos que muchos investigadores han hecho, tanto a nivel nacional como internacional. Ya que el constructivismo se vive, se disfruta y no solo se habla de él. Para hacerlo vivencial es urgente que quienes se desempeñan en la formación de futuros profesionales en Psicopedagogía

2 Conversación con Paulina Godoy (Ministerio de Educación) en el Instituto Profesional de Chile. Año 2012, La Serena. 
hagan vivir la experiencia del aprendizaje, de lo contrario se cae nuevamente en la idea de que la teoría se aleja de la práctica, cuando lo cierto es que a partir de esta, se construye la teoría. Tal como el cartógrafo que dibuja un mapa como aproximación de la realidad, pero que no es más que una representación, es así como psicopedagogas y psicopedagogos -formados bajo un enfoque positivista camuflado y mal maquillado- han ido generando sus aprendizajes. Deben saber cómo la gente aprende, deben aprender a equivocarse, convivir con el error y reorientarlo en la medida que su propensión a enseñar y aprender les diga sobre qué senderos caminar.

El tránsito que ha seguido la Psicopedagogía ha sido caótico. Se ha presentado en muchas ocasiones como la respuesta absoluta a muchas dificultades de aprendizaje que se han podido documentar y clasificar. Ha habitado en paradigmas empírico-positivistas que transmutan las posibilidades de que un estudiante se pueda beneficiar de esta disciplina: que se vean asépticamente. Pulcras y quirúrgicas en el momento de la Intervención Psicopedagógica.

La afirmación de que la Psicopedagogía ha sido caótica se basa en que ha sorteado caminos complejos, pero que a la vez ha simplificado sus procedimientos. Toma ideas de muchas ciencias, las analiza, las compara y las utiliza según sus necesidades. Define muchas veces su carácter de ciencia y centra su objeto de estudio en el aprendizaje en todas sus dimensiones. Vacila entre un terreno y otro, pues asume la incertidumbre como propia, donde todo y nada es posible, donde las ideas se enfocan en la historicidad del estudiantado atendido -acompañado ${ }^{3}$ - y se nutren de las evidencias que se conectan con sus destrezas y habilidades. Es a partir de esto que es considerada ante todo una disciplina científica aplicada por el carácter concreto y particular de su objeto: el proceso educativo, con un núcleo teórico conceptual bien delimitado e integrado por diferentes teorías, principios, categorías y modelos que permiten describir, fundamentar y explicar los fenómenos y procesos que ocurren dentro de dicho objeto, así como diferentes métodos y procedimientos dirigidos a conocer e intervenir para perfeccionar este proceso (Ortiz, 2014)

La Psicopedagogía puede nutrirse de la incertidumbre, debe ser su leit motiv, ya que al existir múltiples barreras de aprendizaje en el contexto escolar, tiene la posibilidad enorme de que esa incertidumbre le dé las herramientas que la pretensión de dominar todos los aspectos intervinientes en el aprender sean utilizados, cuando la realidad dice que cada vez que se aprende, se ignora. Volviendo este proceder, recursivo.

Asumir que estudiantes y profesionales en Psicopedagogía conviven en el saber siempre limitado de una realidad subjetiva y de la ignorancia genérica del aprender, permite reconocer en el otro un ser que es capaz de enseñar. Implica además, un ejercicio de confianza, que va más allá de los aprendizajes escolares y se centra en la persona como constructor de múltiples realidades.

Observando la especialidad en el terreno escolar, tanto la formación de pregrado como el desempeño de profesionales en los colegios, es factible hablar de una Psicopedagogía

3 Se opta por utilizar el término "Acompañamiento Psicopedagógico", ya que el acompañar implica la relación emocional con el otro, a diferencia de lo tradicionalmente usado: "La Intervención Psicopedagógica" donde esta idea contiene a la palabra intervención, que implica rehabilitar un proceso que está mal ejecutado, por lo que se torna invasivo para el estudiante. 
funcional, que se ha transformado en un parche de los fracasos que la escuela ha demostrado generar a lo largo de su historia, confundiendo los espacios de acción efectivos y trabajando bajo la modalidad de los refuerzos pedagógicos, enfatizando en los contenidos no logrados por el estudiantado y que afecta la gestión propia del deber ser de la profesión. Esto hace que un estudiante que no logra hacer el ejercicio de responder como el profesor espera que responda, requiera de más horas de trabajo-poco efectivos muchas veces-dondelas personas profesionales de la Psicopedagogía asisten a los más descendidos en sus aprendizajes, repitiendo en ocasiones las fórmulas que el docente o la docente puso en práctica, centrándose en el contenido y redundando nuevamente en aquella información que al estudiantado no le interesa aprender. Volverse subsidiario de esta práctica es poco productivo, se vuelve a remarcar el error en que la población infantil se encuentra, se asume -consciente o inconscientemente- una actitud de asimetría pedagógica, donde el que sabe es el adulto y el niño o la niña es quien ignora, cuando los hechos indican que todos somos ignorantes. Es necesario invitar a la ignorancia a ser parte de estas conversaciones y de las acciones que de ella surgen; también a la incertidumbre que se viste de mensajera de los aprendizajes que maravillan y encantan, puesto que no hay forma más perenne de aprender que el sorprenderse ante lo nuevo, ante lo diverso; de vivir el quiebre cognitivo y desequilibrarse, donde las certezas se confunden con lo novedoso y posible. Aprender no es más que un acto de entrega mutua, una suma de relaciones que se consensuan y se retroalimentan con el otro, que se vuelve sinérgico, desequilibrante y maravillosamente caótico. Aprender no es repetir, sino que es ir más allá de lo obvio y preguntarse por qué el fuego quema o el viento suena en nuestros oídos, preguntarse por qué nos maravillamos con lo simple que a la vez es complejo. Aprender es un viaje de infinitas estaciones, en un tren que, a cada paso, nos sorprende.

Es así como se opta por una Psicopedagogía que base su ethos en las potencialidades del estudiantado. Descubrir lo que a una persona le es ignorado es aun más complejo que la generación de una evaluación basada en la "certeza" de la estadística ${ }^{4}$. Es necesario triangular la información, pero sobre todo, poder conversar con el otro y poder hacer una aproximación a sus necesidades.

El contenido funciona como germen, como la excusa necesaria para adentrarse en los espacios deseosos de conocimientos, pues la humanidad tiene una propensión innata a aprender. El uso del contenido está en el cómo maravillar a la niñez con ideas novedosas, en cómo hacer que lo cotidiano que es simple, se vuelva complejo. Es, por lo tanto, una forma de emplear una realidad y ponerla en distintas posiciones, logrando que las personas que se educan tomen esa realidad según sus impresiones y la resignifiquen. Y no se trata de que los profesionales en Psicopedagogía sepan de memoria miles de técnicas y estrategias, las cuales dicho sea de paso, cualquiera puede googlear, sino que en el cómo utiliza estas herramientas

4 Ver Taleb, N. (2007) El Cisne Negro. El impacto de lo altamente improbable. Ed. Paidós, España. 
simbólicas. Para ello, es él quien debe también maravillarse y analizar su propio aprender, no quedando en el terreno de lo repetitivo, sino abriendo los senderos a través de la imaginación, dejando de lado por un momento, el exceso de imágenes al optar por la imaginería.

Entonces es necesario explicar que la Psicopedagogía orienta sus acciones hacia las destrezas, habilidades y capacidades del aprender, y eso implica posibilidades infinitas para aprehender relaciones perdurables, posibles todas ellas de ser tomadas. No basta el contenido, ni tampoco la evaluación, sino se tiene en el horizonte la claridad de acompañar psicopedagógicamente a quien aprende, mediando sobre sus posibilidades. Es por lo tanto, trabajo de las y los profesionales de la Psicopedagogía ayudar a la propensión de aprender y enseñar a otros, dar las señales de cómo se hacen los quiebres; en qué momento se acompaña y en qué momento se da autonomía; en qué momento nos hacemos a un lado y dejamos que la persona fluya por sí misma. Esa es la esencia de esta profesión.

\section{La tentación de la certidumbre}

Muchas veces se ha preguntado cuánto tarda el proceso de acompañamiento psicopedagógico -conocido tradicionalmente como intervención- y la verdad es que no existe claridad al respecto, pues los procesos son específicos de cada persona con la que se trabaja. De esta forma y entendiendo que el ser humano es inacabable en su aprender a lo largo de la vida, diría que esto nunca termina, pero se vuelve paradójico desde lo operativo, considerando que difícilmente un profesional podrá estar en todo momento con alguien. Por lo cual, desde el punto de vista práctico, no es posible. Pero sí lo es el poder fijar un horizonte que va desde lo posible a lo probable y desde ahí, a lo realizable ${ }^{5}$, por lo que al avanzar por estos tres estadios se genera la idea de poder armar un trabajo de acompañamiento-mediación psicopedagógica donde se logre hacer una salto cualitativo desde los niveles evidenciados, hasta aquellos que se direccionan como probables de realizar con otro ser humano.

Es así que el marco que propone el decreto 170 la labor de quienes se desempeñan como psicopedagogos y psicopedagogas queda en el ámbito evaluativo. El posterior trabajo de acompañamiento es limitado y bajo ciertas excepciones se permite el ingreso del profesional al aula, ya que se parte de una premisa que está arraigada en la formación inicial, donde se ha asumido que no existe una preparación válida para asumir los desafíos del currículo escolar nacional. Al ser esta una verdad asumida, se pierden oportunidades de educación con el estudiantado que requiere de acompañamientos específicos en las áreas cognitivas, instrumentales y socioafectivas, que van más allá de los conocimientos que puede tener el cuerpo docente, pues la línea de formación es diferente, involucrando múltiples factores del conocer cómo aprendemos los seres humanos.

5 Ver Calvo, C. (2012) Del mapa escolar al territorio educativo. Ed. Universidad de La Serena, Chile. 
Bajo este contexto de evaluación inicial, surge otra pregunta desde las personas adultas, ¿en qué momento se le da el $a l t a^{6}$ al niño? Frente a esta pregunta es necesario mirar el contexto y comprender el lenguaje que se utiliza en la pregunta, asumiendo que el niño "mejorará" frente a la situación clínica del aprendizaje, que en el fondo remite al paradigma empírico-positivista que se ha asociado a la Psicopedagogía como disciplina. En primer lugar, es factible definir un momento, considerando que ese tiempo tiene que ver con la autonomía y madurez que va alcanzando la persona. Mientras mayores acciones de autonomía se van generando, la persona necesita menos del mediador; y en forma inversa, a menor autonomía, mayor será la necesidad que tiene el educando de ser acompañado. Esta acción va de la mano, con dejar que cada persona fluya en sí misma. Que el estudiantado sea capaz de conocer sus espacios válidos de mediación y de conocer otros ámbitos de interés sociocultural, asociados a la eficacia que los mediadores conocidos en su tránsito escolar han impreso en él o ella.

La segunda parte de esta pregunta se relaciona con "dar el alta" y aquí, aparece la enfermedad en forma implícita; el trastorno como nomenclatura psiquiátrica. El hecho de dar el alta, parte desde una concepción patologizante, que luego de un tratamiento adecuado, deja en mejores condiciones al enfermo, que cuando este se inició. En la actualidad, las escuelas han patologizado el aprendizaje, lo han hecho merecedor de una serie de clasificaciones que se alejan de lo realmente importante: la educación como acto de humanidad. Aun cuando el paradigma médico de la Psicopedagogía y sus derivados, datan de inicios del S. XX, se presentan a cada instante. Es necesario reconocer la génesis de la Psicopedagogía, pero eso no implica una entrega y validación absoluta a ella, como si fuese un dogma que hay que seguir al pie de la letra. Por ende, el asumir una Psicopedagogía que aporte desde un terreno vinculante en lo emocional-social presenta posibilidades diferentes en una dinámica que puede alcanzar una (Psico)pedagogía del Bienestar, donde la premisa es educar-se para transformar. Una transformación que transita entre la justicia social y el bienestar emocional.

La patologización del aprendizaje en personas que no presentan alguna barrera de tipo orgánica no es suficiente para dar respuestas a las dificultades que un educando tiene en su escolarización. Si así fuese, habría que preguntarse ¿habrá profesores con necesidades educativas especiales orientadas bajo la concepción del propender a enseñar/aprender? El que un profesor o una profesora conviva en un ambiente con más de cuarenta realidades diferentes, es una complejidad en sí misma, ya que no llegará su mensaje de la misma manera a todos quienes están atentos a sus discursos. Encierra por lo tanto un misterio en el cómo accede cada uno de ellos a lo que el cuerpo docente dice y cómo lo hacen propio y vivencial, no solo como recurso auditivo-visual, sino que significativo.

6 Entendiendo que "dar el alta" es un concepto clínico, se ha optado por esta palabra pues surge en el contexto de las conversaciones cotidianas con algunos padres y madres al referirse al término del proceso de acompañamiento Psicopedagógico, manifestando aquellos usos del lenguaje clínico que han permeado a la educación formal. 
Patologizar el aprendizaje escolar, desde una perspectiva médica es un buen negocio, de hecho se ha convertido en algo tan válido, que cada vez aparecen más y más condiciones-situaciones con una etiqueta nueva (Franco, 2010). Así, el asociar la falta de interés, el desgano, la insatisfacción de las respuestas que el estudiante se ha hecho y que parte del profesorado no ha podido responder, producen como resultado estudiantes que no rinden dentro de la lógica escolar.

\section{Discusión}

Dar el alta es cosificar, delegar responsabilidades y simplificar un proceso que es complejo en sí mismo: el educar(se), pero que en la escuela se vive como escolarización (con todas las connotaciones que esto implica) y que no deja de ser una situación que juega con la semántica, asociándose comúnmente a situaciones sinónimas, cuando la verdad es que ambas distan mucho de parecerse. Son disímiles, pertenecen a dos ámbitos diferentes. La escolarización vive de situaciones repetidas y conocidas; la educación, de la incertidumbre en el descubrimiento de nuevas ideas y aprendizajes.

Un estudiante que ha sido mediado no puede ser dado de alta, pues esto no existe. El educando no es un enfermo que entra al quirófano para ser "tratado" por su enfermedad que es el no aprender los contenidos escolares, sino que es un ser que se nutre de las experiencias que la educación le entrega. Escolarizar y educar son concepciones altamente diferenciables. Por ende, los profesionales en Psicopedagogía deben orientarse hacia esta última, no caer en la simplificación de experiencias clasificatorias superfluas y cargadas de un sentido tramposo, sino que debe propiciar la reflexión para que ese educando que solicita de sus orientaciones, viva la experiencia real de ser, estar y ocurrir en la educación. Es así que el paradigma educativo y epistemológico que podría vivenciar la Psicopedagogía en Chile incluye a elementos de una pedagogía liberadora y vinculante desde lo socioemocional, donde el sujeto es redimido de la opresión a partir de la educación, minimizando las cargas de ignorancia que se asocian al control de aquellos que han visto vulneradas sus oportunidades de aprendizaje formal y que terminan asumiendo una posición de dominación a partir del que sabe más, pues "en los momentos que asumen su liberación, los oprimidos necesitan reconocerse como hombres, en su vocación ontológica e histórica de ser más" (Freire, 2001:45). El saber se constituye como una herramienta de poder y por ende, en este escenario, es factible orientar los esfuerzos de la profesión hacia un camino que no sólo se encargue de aquellos hándicaps que son visualizados en una evaluación estandarizada, sino que le permita a la persona conectarse con un saber que fluye en múltiples direcciones y que tal como el agua va al cántaro, termina por romper los esquemas que han sido configurados en las experiencias de vida que niñas y niños han tenido.

Otro elemento que es necesario integrar corresponde a la Pedagogía Crítica (Victorino, 2006:10) donde "las instituciones escolares son vistas como espacios de lucha y resistencia entre fuerzas de poder ideológicas y culturales por la dominación; es decir, desde la visión de la pedagogía crítica, la escuela no es un espacio neutral, encapsulado y ajeno a conflictos por el 
poder", donde además se asume como "un proceso dialéctico, es la imposibilidad de constituirse como un producto acabado, cerrado o finito", considerando la acción educativa como un terreno que constantemente es fertilizado por la ignorancia.

El enfoque clínico ha logrado mantenerse en el tiempo. Es posible evidenciarlo en el uso del lenguaje, las acciones, la disposición del mobiliario en las aulas, la frontalidad de las clases, la sobrevaloración de lo abstracto por lo concreto, la minimización del juego, entre otros. Hablar de constructivismo es ir más allá de lo evidente. Se trata de leer entre líneas los mensajes que el estudiantado entrega y saber de qué manera se aprende mejor. Hacer constructivismo es minimizar la etiquetación y la tentación de hacer más de lo mismo. Es por ello, que desde el diseño de una sala hasta la mirada de la persona adulta es necesario generar innovaciones. Se intencionan acciones modificantes, tanto en las programaciones de aulas (acompañamientomediación psicopedagógica) como en el uso de material que se dispone para el trabajo, pero más importante aún parece ser el lenguaje interno y externo que lleva a profesionales de la Psicopedagogía a interactuar con niñas y niños que requieren de atenciones con mayores detalles. Es sutil el acercamiento que la persona adulta logra con la niñez en el proceso de aprendizaje-enseñanza para seguir avanzando en el camino que se ha propuesto la profesión.

Hablar de Psicopedagogía implica introducirse en una dinámica versátil sobre el aprender, donde emoción y cognición logren articular sus disposiciones para hacer que el espacio y momento donde ocurre el aprendizaje sea fructífero e ilimitado.

Una (psico)pedagogía liberadora asociada a la pedagogía crítica y del bienestar, requiere un vehículo que transporte sus intenciones mediante los procesos socioafectivos e intelectuales, para indicar en su recorrido que el aprender es una forma de romper esquemas que han sido instalados por el poder a través del saber. Por ende, profesionales de la Psicopedagogía pueden y deben hacer los esfuerzos necesarios para equiparar las condiciones que todo aprendiz debe tener. En el fondo, es un acto de justicia social.

\section{Referencias bibliográficas}

Bejarano, D. (Enero-junio, 2013) El cisne negro. El impacto de lo altamente improbable. Revista Científica Guillermo de Ockham. 11(1), 229-232. Recuperado de http://investigaciones. usbcali.edu.co/ockham/images/volumenes/Volumen11N2/guillermo\%2011-2 c17.pdf

Calvo, C. (2012) Del mapa escolar al territorio educativo. La Serena, Chile: Editorial Universidad de La Serena.

Franco, Y. (2010) Con el DSM-V no va a quedar ninguno sano. El Psicoanalítico, №2, julio de 2010, Argentina. Recuperado de http://www.elpsicoanalitico.com.ar/num2/ep-2.pdf 
Freire, P. (2001) Pedagogía del oprimido, 5º edición. Ed. Siglo XXI

Ministerio de Educación (2009) Normas para determinar los alumnos con necesidades educativas especiales que serán beneficiarios de las subvenciones para educación especial. Recuperado de http://www.leychile.cl/Navegar?idNorma $=1012570$

Ortiz, E.; Mariño, M. (2014) Una comprensión epistemológica de la Psicopedagogía. Cinta de Moebio 49, 22-30. Universidad de Chile, Chile.

Victorino, L. y Lechuga, L. (Noviembre, 2006) Políticas educativas neoliberales y posturas teóricas sociopedagógicas rurales. VII Congreso Latinoamericano de Sociología Rural. Congreso llevado a cabo en Quito, Ecuador. 